\title{
Análisis de la fragmentación de cermets de alúmina-níquel ensayados en Barra Hopkinson a altas velocidades de deformación
}

\author{
F. ORGAZ¹, E. LECUE , A. J. SANCHEZ HERENCIA ${ }^{1}$ Y T. GÓMEZ DEL RÍO ${ }^{2}$ \\ ${ }^{1}$ Instituto de Cerámica y Vidrio, C/ Kelsen, nº 5, 28049 Madrid \\ ${ }^{2}$ Grupo de Durabilidad e Integridad Mecánica de Materiales Estructurales (DIMME). ESCET Universidad Rey Juan Carlos. Madrid.
}

\begin{abstract}
En este trabajo, se presenta un estudio comparativo de la influencia de la velocidad de deformación sobre el comportamiento mecánico a la fragmentación de materiales compuestos de una matriz de alúmina con níquel metálico disperso cuando son sometidos a elevadas velocidades de deformación. La fragmentación bajo cargas dinámicas de compresión a alta velocidad se ha realizado mediante una Barra Hopkinson (SHPB). Se ensayaron materiales compuestos de alúmina-níquel con contenidos en metal de hasta el 50 vol. \%, los cuales fueron procesados coloidalmente a partir de suspensiones acuosas coladas sobre moldes porosos de alúmina. Las muestras se pre-trataron térmicamente para lograr una interfase de unión eficaz y se sinterizaron posteriormente bajo un flujo inerte de atmósfera de argón. Para cada ensayo de fragmentación dinámica se determinaba la correspondiente velocidad de deformación. Las distribuciones estadísticas de tamaños de fragmentos producidos en el impacto con la barra de Hopkinson se determinaron por métodos de tamizado y sus resultados fueron analizados de acuerdo con la función de distribución estadística de Weibull y con el modelo exponencial de Rosin y Rammler. El efecto de la velocidad de deformación sobre el tamaño medio de fragmento se analizó utilizando los modelos de energía existentes. Finalmente, se analizaron los mecanismos de rotura involucrados.
\end{abstract}

Palabras clave: Metal cerámicos, alúmina-níquel, estadística, fragmentación, Barra Hopkinson

Fragmentation analysis of alumina-nickel cermets subjected to Hopkinson bar tests at high strain rates

A comparative study of the influence of the strain rate on the dynamic mechanical behaviour of an alumina matrix with 15 and $50 \%$ of dispersed nickel is presented. The fragmentation under high speed impact compression loads have been studied using a compression split Hopkinson pressure bar (SHPB). Dense alumina and alumina-nickel composites were processed by slip casting of water based slurries on porous moulds. Samples with the metallic phase dispersed were pre-oxidized to achieve an effective joining interface and sintered under flowing inert atmosphere. The strain rate was determined from the impact experiments. The statistics of the SHPB recovered fragments have been determined and analysed according to the exponential models of Weibull and Rosin y Rammler and the effects of the strain rate on the average fragment size are described according to the existing energy models. Finally the rupture mechanisms of the samples and the sources of fracture have been explored and compared to the quasi static mechanical behaviour of these materials.

Keywords: Ceramic-metal composites, alumina-nickel, statistics, fragmentation, Split Hopkinson Pressure Bar (SHPB)

Cómo citar este artículo: Orgaz, F.; Lecue, E.; Sánchez Herencia, A. J. y Gómez del Río, T. (2014): Análisis de la fragmentación de cermets de alúminaníquel ensayados en Barra Hopkinson a altas velocidades de deformación, Bol. Soc. Esp. Ceram. Vidr., 53 (5): 217-226. http://dx.doi.org/10.3989/ cyv. 262014

\section{INTRODUCCIÓN}

El potencial de los materiales de alta alúmina en el campo de la ingeniería es de gran importancia debido a su inercia, alta disponibilidad y su gran resistencia eléctrica y química. Sin embargo, la fragilidad de alúmina limita mucho sus aplicaciones. Uno de los enfoques más prometedores para la mejora de las propiedades mecánicas de los materiales cerámicos son los "cermets" consistentes en incorporar una segunda fase metálica como refuerzo en una matriz cerámica. Una gran variedad de materiales compuestos cerámicos con fases dispersas metálicas se han preparado, sin embargo el sistema $\mathrm{Al}_{2} \mathrm{O}_{3}-\mathrm{Ni}$ ha sido uno de los más estudiados [1-3] debido principalmente a sus posibilidades de alta resistencia a la corrosión y a la oxidación a alta temperatura combinada con una alta resistencia al desgaste y una alta tenacidad a temperatura ambiente. La inclusión de una fase dúctil en una matriz de alúmina rígida mejora el comportamiento mecánico estático a través de la desviación por puenteo o por enroramiento de grieta. Para lograr un refuerzo eficiente, se requiere sin embargo un alto nivel de dispersión de la fase dúctil en la matriz para lograr una alta densidad relativa. Los materiales compuestos de alúmina-níquel se han preparado por varios métodos, incluyendo el procesamiento por sol-gel [4-5], sinterización sin presión, [6-7], la reducción de $\mathrm{NiAl}_{2} \mathrm{O}_{4}$ [8], el prensado en caliente reactivo [9-10] o la reacción de 
aluminio metal con óxidos, incluyendo $\mathrm{Al}_{2} \mathrm{O}_{3}-\mathrm{Ni}$. [11-12]. La sinterización sin presión mediante procesamiento coloidal de metal de $\mathrm{Ni}$ con polvos de óxidos de alúmina se ha utilizado también para preparar varias composiciones diferentes [13-14] habiéndose demostrado que se pueden obtener suspensiones acuosas bien dispersas con dispersantes a base de mezclas de un poli electrolito acrílico de peso molecular 2000 con hidróxido de tetra- metil-amonio a pH 10 [14,15]. Otros autores han estudiado la estabilidad y las propiedades reológicas de las suspensiones de níquel [16-17], donde las superficies de las partículas se enriquecen en $\mathrm{NiO}$ e impiden la disolución de la superficie. El estudio superficial de las suspensiones y su control reológico ha permitido obtener suspensiones concentradas de metales con un $40 \%$ en volumen de sólidos y con densidades en verde tras el colage de un $53 \%$ de la densidad teórica. Sin embargo, hasta la fecha, la mayoría de las propiedades mecánicas estáticas de estos materiales compuestos de alúmina-Ni son aún bajas en comparación con otros compuestos de metal de cerámica debido a la porosidad y a la falta de adhesión en la interfaz metal-cerámica. Estas bajas prestaciones mecánicas han sido atribuidas a la falta de adherencia en la interfase metal-cerámica y a la porosidad haciendo necesario la formación de una pequeña cantidad de $\mathrm{NiAl}_{2} \mathrm{O}_{4}$ en la interfaz de $\mathrm{Ni}-\mathrm{Al}_{2} \mathrm{O}_{3}$ para promover una buena adhesión [18]. Solamente por prensado en caliente reactivo se ha conseguido una microestructura inter- penetrante capaz de producir una alta resistencia mecánica $(600 \mathrm{MPa})$ y una alta tenacidad (12 $\mathrm{MPa} \sqrt{\mathrm{m}})$ [19].

Aun cuando el comportamiento mecánico cuasi-estático de estos materiales ha sido estudiado, no existen estudios tendentes a explorar el comportamiento mecánico de estos materiales cuando son sometidos a altas velocidades de deformación. Esto es importante para un diseño eficiente de sistemas que intenten utilizar estos materiales en aplicaciones estructurales donde estén sometidos a altas velocidades de deformación. Es vital para el progreso continuo de estos materiales compuestos, conocer los mecanismos denucleación, crecimiento y coalescencia de grietas que determinan el deterioro de estos materiales compuestos cuando son sometidos a escenarios de carga de altas velocidades de deformación, diseñar estrategias de mitigación de daños y establecer relaciones entre parámetros constitutivos y velocidades de deformación. El avance en el conocimiento sobre la fractura en condiciones dinámicas de materiales se basa en el empleo de diferentes técnicas instrumentales y sofisticadas herramientas de diagnóstico y evaluación [20]. La barra Hopkinson (SHPB) ha sido utilizada recientemente para analizar el comportamiento a compresión y fractura dinámica de materiales cerámicos y materiales compuestos a altas velocidades de deformación. Modificaciones de la técnica convencional se han realizado mediante el empleo de las técnicas de modulación de impulsos desarrollados por Frew et al. [21] y los sistemas de control de carga de impacto único con sistemas mecánicos de atrapamiento de momentos desarrollados por Nemat-Nasser et al. [22]. En los últimos años se han publicado un relativamente elevado número de trabajos sobre el comportamiento mecánico de materiales cerámicos y compuestos sometidos a altas velocidades de deformación, las cuales han sido revisadas recientemente por Subhash et al. [23]. Los resultados experimentales muestran en general un aumento aparente de la resistencia y de la tenacidad a altas velocidades de deformación por encima de un límite aproximado de $200 \mathrm{~s}^{-1}$. En este punto, se produce una transición donde aparece un régimen de aumento de la resistencia y reducción en el tamaño de fragmento con la velocidad de deformación, cuyos mecanismos responsables han sido descritos por Lankford [24, 25], siendo la interpretación más aceptada la basada en la inercia de grieta como un factor clave que controla la transición hacia el régimen altamente sensible de la fractura dinámica de los sólidos frágiles con la velocidad de deformación. Tres mecanismos o fuentes de daño han sido considerados en la literatura como causantes del proceso de fractura dinámica y fragmentación: a) En caso de no confinamiento exterior, la fragmentación se produce por un proceso de nucleación, crecimiento y coalescencia final de defectos y grietas inducidas en el proceso de fabricación que conducen a un desencadenamiento de fractura múltiple con separación axial seguido de fragmentación columnar consecuencia de la compresión máxima axial, b) mecanismos de cizalla localizada que produce flujo plástico en el material por la presencia de una presión de confinamiento lateral y c) procesos de flujo plástico por una restricción inercial que produce micro-grietas inducidas por apilamiento de dislocaciones. Así, cerámicas como $\mathrm{Al}_{2} \mathrm{O}_{3}$ y $\mathrm{Si}_{3} \mathrm{~N}_{4}$ pueden experimentar flujo plástico por deslizamiento múltiple bajo carga de compresión cuando son sometidas a altas velocidades de deformación cercanas al límite elástico de Hugoniot y si existen restricciones de inercia suficientes (ausencia de defectos de procesamiento y tamaños de grano pequeño) para evitar la nucleación, crecimiento y coalescencia de grietas que conduce a la fragmentación típica dinámica inducida por fisuras inherentes en el material [25]. La mayor parte de los estudios teóricos sobre la fragmentación dinámica de cerámicas y sólidos frágiles se orientan a explicar e interpretar el aumento de resistencia con velocidad de deformación, a explicar los mecanismos de fractura dinámica y a proponer diversas teorías y modelos para predecir la distribución de tamaños de fragmentos y sus correspondientes parámetros de escala (tamaño medio de fragmento), así como modelos predictivos capaces de establecer las relaciones cuantitativas entre velocidad de deformación y parámetros de escala. Todas ellas, pueden ser organizadas en cuatro grupos a) teoría probabilística basada en estadísticas-geométricas de la fragmentación [2627], b) modelos de balance de energía de la fragmentación [28-30], c) modelos de zona cohesiva [31-33] y d) enfoques computacionales 3D [34]. Otros enfoques basados en la mecánica de fractura han sido también desarrollados [23].

El objetivo del presente trabajo es analizar el comportamiento a la fragmentación de materiales compuestos de alúmina- níquel, procesados coloidalmente, y sometidos a altas velocidades de deformación con una Barra Hopkinson. A partir de los ensayos se ha determinado el comportamiento estadístico de los fragmentos generados por las muestras tras el ensayo y las relaciones entre los tamaños medio de fragmento y la velocidad de deformación del ensayo. Los resultados son discutidos en base a su consistencia con resultados y experiencias previas en otros materiales y con los modelos teóricos explicativos existentes. De este análisis también se ha estudiado por microscopía electrónica la superficie de los fragmentos. Se pretende extraer relevante información sobre los mecanismos de fractura dinámica y la estadística de fragmentación de estos materiales. 


\section{PARTE EXPERIMENTAL}

\subsection{Materiales}

Materiales compuestos de alúmina-níquel con contenidos de metal hasta un 50 vol. \% se procesaron coloidalmente por colado de suspensiones acuosas sobre moldes porosos de alúmina. Se emplearon los siguientes polvos comerciales: níquel T110, Inco, Canadá con un tamaño medio de partícula de $2,5 \mu \mathrm{m}$ y alúmina Condea. HPA05, USA con un tamaño medio de partícula de $0,35 \mu \mathrm{m}$. Las diferentes suspensiones acuosas de níquel y alúmina puros con contenido en sólidos entre 15 y $50 \%$ en volumen se prepararon empleando como agente dispersante Duramax D3005, el cual era agregado a la suspensión en un porcentaje del $1 \%$ en peso, referido a sólidos. A esta concentración las suspensiones mostraban la máxima fluidez. Los sólidos se dispersaron empleando una sonda de ultrasonidos de 400 watios (Dr. Hielscher US400) con agitación mecánica. Las suspensiones de metal y cerámicas puras se mezclaron en las proporciones adecuadas aplicando de nuevo ultrasonidos y agitación mecánica durante al menos una hora antes de colar. En trabajos anteriores se puede encontrar más detalle del procesamiento de estos materiales [15]. El colage se realizó sobre sustratos porosos de alúmina sinterizada preparados en el laboratorio. Se colaron cilindros con un diámetro de aproximadamente $6 \mathrm{~mm}$ de diámetro y $15 \mathrm{~mm}$ de altura los cuales fueron secados a temperatura ambiente durante 24 horas. Las muestras secas fueron pretratadas en un horno a $300{ }^{\circ} \mathrm{C}$ durante 2 horas para lograr una interfaz de unión metal-cerámico y a continuación se sinterizaban en un horno convencional sin presión a $1450{ }^{\circ} \mathrm{C}$ durante $2 \mathrm{~h}$ bajo un flujo de atmósfera inerte de argón. Los discos sinterizados fueron posteriormente cortados y mecanizados con el fin de obtener cilindros de entre $7 \mathrm{~mm}$ de largo y $6 \mathrm{~mm}$ de diámetro para las medidas de resistencia a la fractura dinámica. Las microestructuras de las muestras cocidas se estudiaron, sobre las superficies pulidas con polvo de diamante $1 \mu \mathrm{m}$, por microscopía óptica (Leica, modelo DMR) y microscopía electrónica de barrido (Karl-Zeiss, el DSM-950 modelo). La Tabla I muestra las propiedades físicas y mecánicas de los materiales compuestos estudiados. La densidad se determinó por el método de inmersión, la tenacidad mediante el método de indentación, la dureza por indentación Vickers y el módulo de Young a partir de la pendiente de la curva tensión -deformación obtenida en ensayos cuasi estáticos realizados en una máquina Instron.

TAbla I. Propiedades físicAs Y MECÁNICAS DE MATERIALES COMPUESTOS METAL-CERÁMICOS DE ALÚMINA-NÍQUEL

\begin{tabular}{|c|c|c|c|c|c|}
\hline & $\begin{array}{c}\text { Densidad } \\
\left(\mathbf{g} / \mathbf{c m}^{3}\right)\end{array}$ & $\begin{array}{c}\text { Porosidad } \\
(\%)\end{array}$ & $\begin{array}{c}\text { Tenacidad } \\
\mathbf{K}_{\text {ic }}(\mathbf{M P a} \\
\sqrt{\mathbf{m m}})\end{array}$ & $\begin{array}{c}\text { Dureza } \\
(\mathbf{G P a})\end{array}$ & $\begin{array}{c}\text { Módulo } \\
\mathbf{d e} \\
\text { Young } \\
(\mathbf{G P a})\end{array}$ \\
\hline Alúmina & 3,87 & 2,5 & 2,79 & 4,57 & 380 \\
\hline $\begin{array}{c}\text { Alumina } \\
+15 \% \\
\text { vol. Ni }\end{array}$ & 4,46 & 4,84 & 3,76 & 2,9 & 335 \\
\hline $\begin{array}{c}\text { Alumina } \\
+50 \% \\
\text { vol. Ni }\end{array}$ & 4,8 & 24,5 & 4,1 & 1,42 & 170 \\
\hline
\end{tabular}

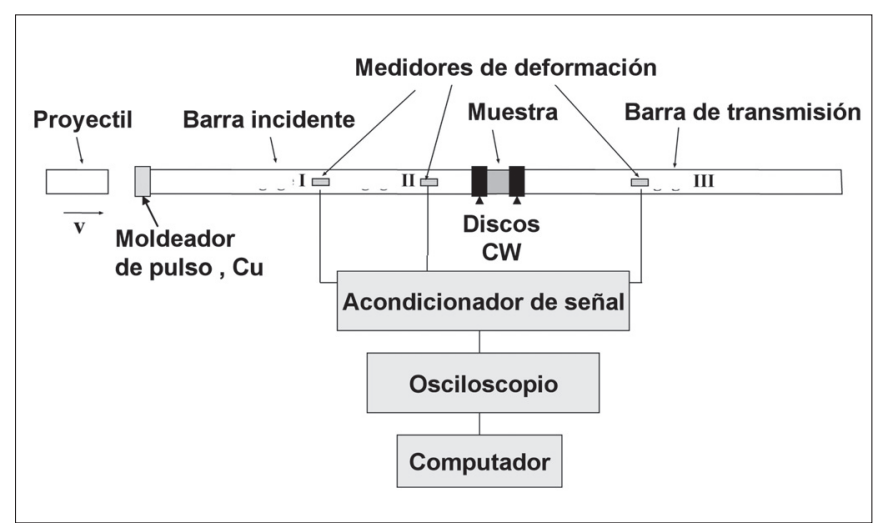

Figura 1. Representación esquemática de una barra de Hopkinson (SHPB).

\subsection{Ensayos experimentales con Barra Hopkinson}

Los ensayos de compresión dinámica se realizaron utilizando una barra Hopkinson (20,35). Este equipo experimental permite el análisis de la respuesta del material bajo un estado uniaxial de compresión a altas velocidades de deformación. Consiste básicamente (Figura 1) en un cañón de gas, un proyectil, una barra incidente y una barra transmisora, además, de los soportes y el sistema de adquisición de datos. A través de este sistema se pueden determinar los pulsos incidente, reflejado y transmitido en las barras mediante bandas extensométricas pegadas en las mismas. Las señales de bandas extenso-métricas se registran utilizando un acondicionador VISHAY 2200 junto con un osciloscopio digital TEKTRONIX TDS 420A. Para evitar el deterioro de las barras se colocaron a ambos lados de la muestra, y en contacto con las barras, dos discos de alta rigidez, y similar impedancia mecánica, de carburo de tungsteno (CW $3 \%$ Co) de $12 \mathrm{~mm}$ de diámetro y $8 \mathrm{~mm}$ de espesor. También se colocaron discos de cobre recocido de $10 \mathrm{~mm}$ de diámetro y $5 \mathrm{~mm}$ espesor como modeladores de pulso, delante de la barra incidente. Con esto se pretendía controlar los perfiles de carga y descarga del pulso incidente y asegurar así unas mejores condiciones de ensayo, equilibrio en la muestra y velocidad de deformación constante durante el ensayo. Las muestras usadas para las medidas de compresión dinámica se mantenían en posición axial a fin de evitar inclinaciones y generación de posibles tensiones de cortadura. Asumiendo que ambas barras se comportan elásticamente, y que existe equilibrio durante el ensayo, las medidas de deformación sobre las barras proporcionan la tensión y deformación de la muestra de acuerdo a las siguientes expresiones [36-37]:

$$
\begin{aligned}
& \sigma_{\mathrm{p}}(\mathrm{t})=\mathrm{E}\left(\mathrm{A}_{\mathrm{b}} / \mathrm{A}_{\mathrm{p}}\right) \varepsilon_{\mathrm{T}}(\mathrm{t}) \\
& \dot{\varepsilon}(\mathrm{t})=-\left(2 \mathrm{c} / \mathrm{L}_{0}\right) \varepsilon_{\mathrm{R}}(\mathrm{t}) \\
& \varepsilon_{\mathrm{p}}(\mathrm{t})=\int_{0}^{\mathrm{t}} \dot{\varepsilon} \mathrm{dt}
\end{aligned}
$$

donde $\sigma_{\mathrm{p}}(\mathrm{t}) \dot{\varepsilon}(\mathrm{t})$ y $\varepsilon_{\mathrm{p}}(\mathrm{t})$ son la tensión, la velocidad de deformación y la deformación en la muestra, respectivamente; 
$\varepsilon_{\mathrm{R}}(\mathrm{t}) \varepsilon_{\mathrm{T}}(\mathrm{t})$ y $\varepsilon_{\mathrm{S}}(\mathrm{t})$ son las deformaciones producidas por la onda reflejada, transmitida e incidente respectivamente, $\dot{\varepsilon}$ la velocidad de deformación; $A_{p} \mathrm{y} \mathrm{L}_{\mathrm{p}}$ son la sección y longitud de las muestras, y $A_{b^{\prime}} E$ y c $=\sqrt{E} / \rho$ son la sección, módulo de Young, densidad y velocidad de propagación de las ondas elásticas en las barras respectivamente. Las velocidades de deformación oscilaban entre $800-7500 \mathrm{~s}^{-1}$. Sobre la base de los cálculos unidimensionales $(36,37)$ se ha establecido que la amplitud del pulso transmitido es una medida de la tensión en la muestra y la amplitud del impulso reflejado es proporcional a la velocidad de deformación, é, en la muestra, Integrando la velocidad de deformación con respecto al tiempo da la deformación axial en la muestra. Los experimentos fueron realizados de tal manera que se cumplieran siempre los supuestos o hipótesis principales sobre los que se sustenta las ecuaciones anteriores. Una de las hipótesis necesarias para la validez de los ensayos de barra Hopkinson, es que haya un equilibrio cuasiestático en la probeta durante la mayor parte del ensayo. Se ha comprobado que el material alcanza el equilibrio rápidamente y que el ensayo puede considerarse cuasiestático, pese a la alta velocidad de deformación alcanzada. Para ello se han comparado las ondas incidente y reflejada (en un extremo de la probeta) con la onda transmitida (extremo opuesto de la probeta) y se ha comprobado que existe un equilibrio tensional pasados los primeros $10 \mu \mathrm{s}$ del ensayo, aproximadamente. Otra hipótesis del ensayo en barra Hopkinson es la propagación de ondas elásticas unidimensionalmente en las barras del equipo donde el estado tensional debe ser unidimensional y axialmente uniforme; de este modo, se pueden determinar las fuerzas aplicadas y los desplazamientos producidos en los extremos de las barras en contacto con la probeta, componiendo los efectos de las ondas incidente, reflejada y transmitida. Igualmente era siempre observado que las señales registradas en las galgas extensiométricas correspondían a las verdaderas medidas de la respuesta de las muestras, especialmente en un período muy corto (dispersión mínima).

\subsection{Recuperación y análisis de fragmentos}

El análisis post-mortem de las muestras fragmentadas después de haber sido ensayadas con la barra Hopkinson, se realizó mediante técnicas de tamizado de polvos. Los fragmentos eran recogidos en el entorno de la muestra a ensayar mediante bolsas de plástico. La distribución de los tamaños de los fragmentos se caracterizó en función de su capacidad o incapacidad para pasar a través de una serie de tamices de diferente grado de abertura mientras eran sometidos a vibración. Se utilizaron tamices con mallas entre 50 y 2.500 micras. Después de tamizado por diferentes mallas, se medía la cantidad de masa (peso) para cada tamaño de fragmento (malla) así como la distribución simple y la correspondiente masa acumulada de fragmentos de un tamaño superior o inferior a un tamaño determinado. Para las muestras analizadas, la masa total de los fragmentos era alrededor de $M_{T} \approx 0,200$ g. Todos los fragmentos se considerados esféricos debido a que la precisión de análisis de tamizado depende en cierta medida de la relación de aspecto de la partícula, si bien por el tipo de fractura los más grandes tenían aspecto columnar.

\section{RESULTADOS EXPERIMENTALES Y DISCUSION}

\subsection{Microestructura}

La Figura 2 muestra las micrografías ópticas de la superficie pulida de los diferentes materiales compuestos de alúmina/ níquel. Las fases más oscuras y brillantes son los granos de alúmina y níquel, respectivamente. Como puede apreciarse, las partículas metálicas se encuentran uniformemente dispersas en la matriz de alúmina manteniendo mayoritariamente el tamaño de partícula inicial. Se observa la presencia de algunos aglomerados consecuencia de los altos contenidos de la segunda fase. La fase metálica se encuentra fuertemente unida a la matriz cerámica, aunque se puede apreciar bien la presencia de una porosidad irregular (de color negro en las micrografías) o hendiduras de arranque del material surgidas en la fase de pulido.

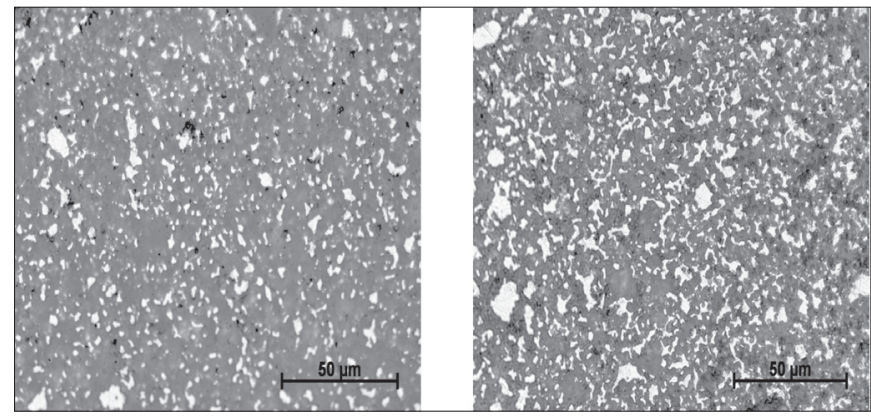

Figura 2. Microscopía óptica de los diferentes composites alúmina- níquel: a) $15 \%$ volumen $\mathrm{Ni} \mathrm{y} \mathrm{b)} 50 \%$ volumen $\mathrm{Ni}$.

\subsection{Análisis estadístico de la fragmentación dinámica}

\subsubsection{MODELOS DE DISTRIBUCIÓN ESTADÍSTICA DE FRAGMENTOS. ESTADO DEL ARTE}

Diferentes distribuciones exponenciales o de tipo exponencial (gamma, Weibull, Voronoi) y leyes de potencia han sido propuestas en la literatura para describir la naturaleza estadística de fragmentos [39]. Lienau [40] asume que el proceso de fragmentación se inicia desde fisuras distribuidas al azar sobre una línea, y la distribución de longitudes de fragmentos está determinada por las distancias entre fisuras de tal manera que la distribución en el número de fragmentos mayores que una longitud 1 es una exponencial de la longitud de dicho fragmento

$\mathrm{F}(\mathrm{x})=1-\exp (-\mathrm{x} \mu)$

donde $\lambda=1 / \mu$, siendo $\lambda$ el parámetro de escala o escala de longitud.

Esta caracterización de los resultados estadísticos del proceso de fragmentación, ha sido extendida a dos y tres dimensiones por Grady and Kipp [41, 42] Específicamente la relación tridimensional es

$\mathrm{N}(\mathrm{m})=\mathrm{N}_{0} \exp \left(-\mathrm{N}_{0} \mathrm{~m}\right)$

donde $\mathrm{N}(\mathrm{m})$ es el número acumulativo de fragmentos con masa $>\mathrm{m}, \mathrm{N}_{0}$ el número total de fragmentos y $\mathrm{m}$ la masa de cada fragmento. Mott [26] propuso también una teoría de la 
fragmentación basada en la estadística geométrica sobre la base de la creación aleatoria de grietas y su interacción a través de ondas de descarga, para explicar la distribución de tamaño de fragmentos de tipo exponencial observada en anillos metálicos cuando eran sometidos a ensayos de expansión dinámica. Esta teoría probabilística, aplicable a la rotura de metales, permite predecir, para determinado tipo de material al que se le somete a una carga expansiva de alta velocidad de deformación, la distribución de tamaño de los fragmentos y los correspondientes parámetros de escala característicos. De acuerdo con esta teoría, Mott y Linfoot [43] descubrieron en sus experimentos que el número acumulado de fragmentos con masa $>$ m puede ser mejor descrita por

$\mathrm{N}(\mathrm{m})=\mathrm{N}_{0} \exp \left[-(3 \mathrm{No} \mathrm{m})^{1 / 3}\right]$

La cual, extendida a dos dimensiones da:

$\mathrm{N}(\mathrm{a})=\mathrm{N}_{0} \exp \left[(-2 \mu \mathrm{a})^{1 / 2}\right]$

donde $\mathrm{N}_{0}$ es el número total de fragmentos, a es el área del fragmento, y $\mu$ es un parámetro de ajuste dado por $\mathrm{CN}_{0}=\mathrm{A}$ con A denota el área total de fragmentos y $\mathrm{C}$ una constante. Grady and Kipp [40] por su parte asumen que la probabilidad de fractura es uniforme espacialmente, que todos los puntos del cuerpo son accesibles a la fractura y que los lugares de fractura pueden ser arbitrariamente unidos unos con otros. Por consiguiente, un evento puede ser considerado como un sistema de fractura continua si el tamaño medio de fragmento es grande en relación al tamaño de fragmento mínimo. Bajo estas condiciones, propusieron la siguiente función de distribución exponencial para la distribución de tamaño de fragmentos [44]

$\left.\mathrm{N}(\mathrm{m})=\mathrm{N}_{0} \exp [\mathrm{M} / \mu-1] \operatorname{Ln}[(1-\mathrm{m}) / \mathrm{M})\right]$

donde $\mathrm{N}$ es el número acumulado de fragmentos con masa $>\mathrm{m}$, M es la masa total y $\mu$ la masa media de fragmento.

Rosin y Rammler [27], aplicando técnicas de tamizado, utilizaron una distribución tipo Weibull para su análisis estadístico de fragmentos, proponiendo tambien una función exponencial para describir las distribuciones de tamaño de fragmentos de carbón en función de su tamaño. Esta distribución se expresa comúnmente como una fracción de masa acumulativa (CFM) de la masa total de la muestra de $\mathrm{M}$ T que es mayor que un tamaño de $\mathrm{x},(\mathrm{M}>\mathrm{x})$, la cual viene dada por la expresión:

$1-\left[\mathrm{M}(<\mathrm{x}) / \mathrm{M}_{\mathrm{T}}\right]=\left[\mathrm{M}(>\mathrm{x}) / \mathrm{M}_{\mathrm{T}}\right]=\exp \left[-(\mathrm{x} / \lambda)^{\mathrm{n}}\right]$

donde $\lambda$ es el parámetro o longitud de escala de la distribución y $\mathrm{n}$ el parámetro de forma cuyos valores oscilan usualmente entre 0,5, 1 y 1, 5. Dicha ecuación puede expresarse también en la forma de fracción de masa acumulativa que es menor que un tamaño $\mathrm{x}(\mathrm{M}<\mathrm{x})$ mediante:

$\operatorname{CFM}(<x)=\left[M(<x) / M_{T}\right]=1-\exp \left[-(x / \lambda)^{n}\right]$

Tomando logaritmos de ecuación (9) se obtiene la expresión

$\operatorname{Ln}\left[M(>x) / M_{T}\right]=-(x / \lambda)^{n}$

La ecuación (9) expuesta en forma doble logarítmica de Weibull viene dada por una línea recta de pendiente $\mathrm{m}$ (parámetro de forma)

$\operatorname{Ln}\left[-\operatorname{Ln}\left[\mathrm{M}(>\mathrm{x}) / \mathrm{M}_{\mathrm{T}}\right]\right]=\mathrm{n} \operatorname{Ln}(\mathrm{x} / \lambda)$

Estas técnicas de análisis estadístico de la masa acumulada de fragmentos (CFM) frente a tamaño de fragmento, son más útiles y fáciles de aplicar que las representaciones anteriores de Mot.-Linfoot [26,43] y de Grady [41,42] dadas por las ecuaciones (5), (6) y (7), sobre todo en los fragmentos muy finos, debido a las dificultades que estas últimas conllevan en el conteo del número y en el peso de cada fragmento (masa) o área de cada uno de los fragmentos individuales. Por otro lado, la teoría de Mott Mott and Linfoot no es de aplicación a este tipo de materiales frágiles ya que es solamente aplicable a materiales que expanden de forma dúctil antes de producirse la fractura.

\subsubsection{RESULTADOS EXPERIMENTALES \\ DE FRAGMENTACIÓN ESTADÍSTICA}

Las distribuciones estadísticas de fragmentos recuperados de la muestra alúmina pura y del material metal cerámico 85Alumia/15Ni sometidos a varias velocidades de deformación se muestran en la Figura 3 donde se representa el peso acumulativo de fragmentos mayor que un tamaño dado en función del tamaño de dichos fragmentos, $x$, para los diferentes ensayos, $\mathrm{Ti}$, de muestras metal-cerámicas sometidas a diferentes velocidades de deformación. Como puede apreciarse, los fragmentos más grandes fueron recuperados de tasas de deformación más bajas, lo cual puede ser explicado cómo debido a una menor energía involucrados en el proceso de fragmentación. El tamaño medio de fragmento era calculado a partir del valor en el que el peso acumulado de los fragmentos superior a $x$ es de $50 \%$ [38].

La Figura 4 muestra una representación de los resultados experimentales de fragmentación acuerdo a la representación doble logarítmica de Weibull (ecuación 12) de diferentes ensayos realizados sobre materiales de $85 \%$ Alumina- $15 \%$ Níquel. Puede observarse como la distribución de tamaño de fragmentos sigue una distribución tipo Weibull con un parámetro de forma de $m$ entre 1,3 y 1,5. La figura 5 muestra la cuantificación de la distribución del tamaño de fragmento de los resultados anteriores ajustados a la ecuación de Rosin y Rammler para un parámetro de forma $\mathrm{n}=1,5$ donde logaritmo neperiano de la masa acumulada de los fragmentos $>\mathrm{x}$ se representa frente al tamaño de fragmento, $x^{n}$, siendo $n$ el 


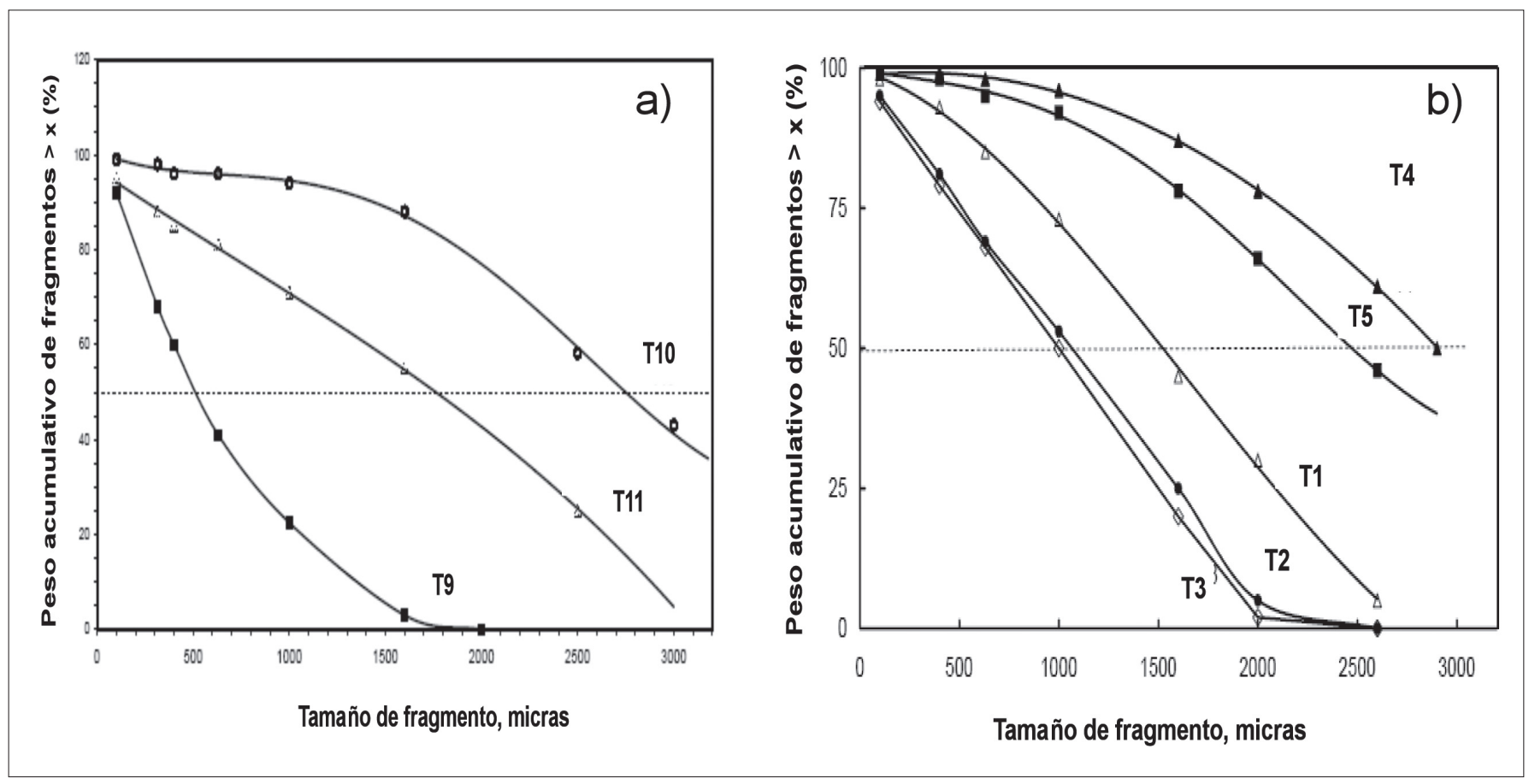

Figura 3. Peso acumulativo de fragmentos con tamaño $>$ s para a) alúmina b) una muestra de $85 \%$ alúmina $-15 \%$ Ni sometida a diferentes test de velocidad de deformación Ti (T9=1200 s-1; T11=40 s-1; T10 $\left.=10 \mathrm{~s}-1 ; \mathrm{T} 4=36 \mathrm{~s}^{-1 ;} \mathrm{T} 5=62 \mathrm{~s}^{-1 ;} \mathrm{T} 1=435 \mathrm{~s}^{-1} ; \mathrm{T} 2=550 \mathrm{~s}^{-1} \mathrm{y} \mathrm{T}^{-1}=655 \mathrm{~s}^{-1}\right)$.

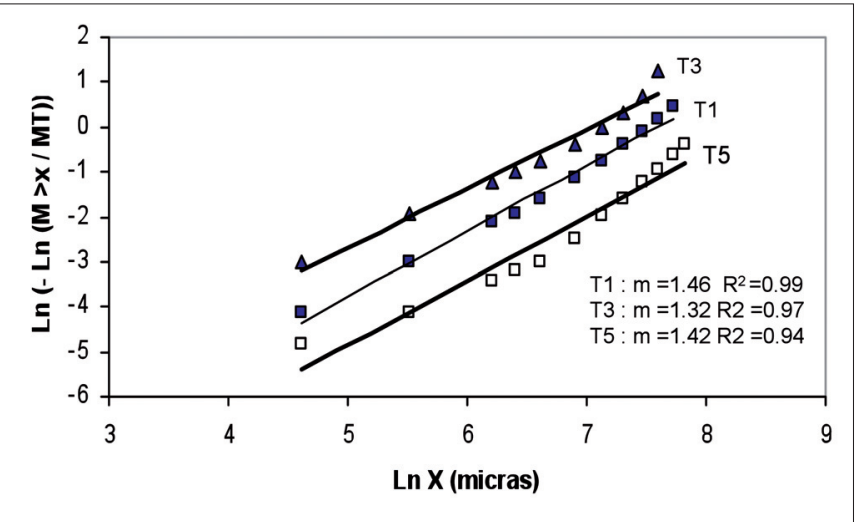

Figura 4. Distribución de tamaño de fragmentos de muestras ensayadas, ajustados a una distribución de tipo Weibull para ensayos Ti realizados a diferentes velocidades de deformación $\varepsilon^{\prime}\left(\mathrm{T} 3=654 \mathrm{~s}^{-1}\right.$; T1 $\left.435 \mathrm{~s}^{-1} \mathrm{~T} 5=65 \mathrm{~s}^{-1}\right)$.

correspondiente parámetro de forma. Como puede apreciarse los resultados pueden aproximarse a una relación bilineal y por tanto a dos regímenes de fragmentación, cada uno con diferentes parámetros de escalas $\lambda_{1}$ y $\lambda_{2}$ correspondientes a la fracción fina y gruesa respectivamente de la distribución. Estos resultados son consistentes con la teoría de la fragmentación de no- equilibrio desarrollada por Grady [39] para materiales frágiles, que considera que los materiales frágiles experimentan su inicio de rotura y posterior fragmentación a una energía de deformación elástica que es superior a la predicha por métodos cuasi estáticos, debido fundamentalmente a un proceso de acumulación de energía. Este proceso de no equilibrio (dependencia del tiempo) típico de los materiales frágiles da lugar a la aparición de dos parámetros de escala $\lambda_{\mathrm{e}}$ (tamaño de fragmento fino) y $\lambda_{C}$ (tamaño de fragmento grande) que son los que gobiernan el proceso de fragmentación fruto de una rotura, ya que las fracturas al nivel de $\lambda_{C}$ no son capaces de disipar toda

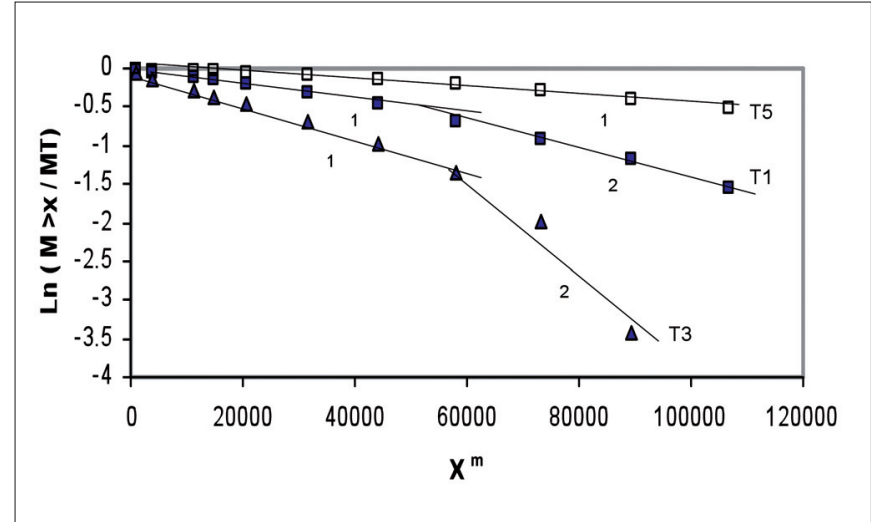

Figura 5. Logaritmo neperiano del peso acumulativo de fragmentos frente al tamaño medio de fragmento elevado a $1.5\left(\mathrm{x} / \lambda^{\mathrm{m}}\right.$ para composites de $85 \%$ alúmina- $15 \% \mathrm{Ni}$ sometidos a diferentes velocidades de deformación, de acuerdo a ecuación 10.

la energía almacenada en el material, dando lugar a un proceso de rotura en cascada y secuenciación y consiguientemente a la aparición de un segundo parámetro de escala. La existencia de dos regímenes de fragmentación se corresponde también con las diferentes regiones, zonas de deterioro o patrones de fragmentación observados por varios autores en ensayos de impactos a alta velocidad [20] donde aparece una región pulverulenta y una región exterior agrietada llena de fisuras y de tipo radial.

\subsection{Análisis de las relaciones entre velocidad de deformación y tamaño de fragmento.}

\subsubsection{TEORÍAS Y MODELOS DESCRIPTIVOS}

Varias teorías y modelos han sido propuestos para describir las relaciones entre velocidad de deformación y tamaño de fragmento pudiendo ser agrupados en modelos 
energéticos $(28,29,30)$, y modelos cohesivos y computacionales (31-33). Todos ellos analizan las tendencias de la rotura dinámica y la fragmentación de los materiales en función de sus propiedades constitutivas, de sus formas geométricas y de las condiciones de carga aplicadas. Así, Grady [28] y Kipp y Grady [29] propusieron una teoría energética de la fragmentación para analizar las relaciones entre la velocidad de deformación y el tamaño medio de fragmento resultante del proceso de fragmentación. Asumiendo que la energía cinética local es convertida en la energía necesaria para crear nuevas superficies, el tamaño medio de fragmento puede ser calculado según la expresión

$\mathrm{L}=24^{1 / 3}\left(\mathrm{~K}_{\mathrm{IC}} / \rho C \varepsilon \dot{\varepsilon}\right)^{2 / 3}$

donde $\mathrm{K}_{\mathrm{IC}} \rho$ y c son respectivamente la energía de fractura del material (factor crítico de intensidad de tensión modo I), la densidad y la velocidad de la onda longitudinal. Esta teoría predice que el tamaño medio de fragmento se escala con la velocidad de deformación por una relación $\mathrm{L} \approx \varepsilon^{-2 / 3}$. Este modelo fue modificado por Glenn and Chudnovksy (GC) [30] quienes propusieron un nuevo modelo energético para calcular el tamaño medio de fragmento en función de la velocidad de deformación, introduciendo un término de corrección en el modelo de Grady que contempla la energía elástica de deformación almacenada antes de la fractura así como la existencia de un pico de tensión $\sigma_{\text {máx. }}$ a partir del cual se inicia la fragmentación (umbral de tensión $\sigma_{\text {máx }}$ para la fragmentación). En este modelo el tamaño medio de fragmento viene dado por la expresión

$\mathrm{s}=4 \sqrt{(}(\alpha / 3) \cdot \operatorname{senh}(\phi / 3)$

$$
\begin{array}{r}
\text { Donde } \operatorname{senh}\left(\frac{\Phi}{3}\right)=\frac{e^{\Phi / 3}-e^{\Phi / 3}}{2}=\frac{e^{2 \Phi / 3}-\mathbf{1}}{2 e^{\Phi / 3}}, \text { siendo } \\
\varphi=\operatorname{senh}^{-1}\left[\boldsymbol{\beta}\left(\frac{3}{\alpha}\right)^{3 / 2}\right] ; \alpha=\frac{3 \sigma_{c}^{2}}{\rho \mathbf{E} \varepsilon^{2}} \text { y } \beta=\frac{3 G c}{2 \rho \varepsilon^{2}}, \text { E es }
\end{array}
$$

el módulo de Young y $\sigma_{c}$ es la resistencia compresiva del material antes de la fractura.

Como ha sido reseñado con anterioridad, el modelo de Grady [28] asume que la energía cinética local debe ser balanceada por la energía requerida para crear nuevas superficies. Esta predicción resulta correcta cuando se reduce el tamaño de fragmento al aumentar la velocidad de deformación [32-33]. El modelo de Glenn and Chudnovsky permite por otra parte capturar mejor las distribuciones de fragmentos a bajas velocidades de deformación ya que se produce un retraso asociado en el proceso de fragmentación consecuencia de la no despreciable acumulación de energía elástica de deformación en la muestra. Las predicciones de ambos modelos son similares a altas velocidades de deformación donde la energía cinética local es dominante, mientras que en la región de muy baja velocidad de deformación (cuasi estático) la teoría de Glenn and Chudnovsky predice un tamaño medio de fragmento independiente de la velocidad de deformación, el cual viene dado por $X=\frac{2 E G_{c}}{\sigma_{c}^{2}}$ donde E es el módulo de Young y $\sigma_{c}$ es

la resistencia compresiva del material antes de la fractura o el valor crítico al cual el material tensionado es fragmentado. Ambos modelos energéticos han sido utilizados ampliamente en la literatura para analizar los procesos de fractura en función de la velocidad de deformación.

\subsubsection{RESULTADOS EXPERIMENTALES Y DISCUSIÓN DE LA INFLUENCIA DE LA VELOCIDAD DE DEFORMACIÓN SOBRE EL TAMAÑO MEDIO DE FRAGMENTO}

La figura 6 muestra el efecto de la velocidad de deformación sobre el tamaño medio de fragmento. Puede apreciarse una relación lineal entre logaritmo neperiano de tamaño medio de fragmento y logaritmo neperiano de velocidad de deformación de tal manera que se produce una reducción del tamaño de fragmento medio con el aumento de la velocidad de deformación. Se observa también en dicha grafica, de acuerdo con ecuación 13, un desplazamiento a tamaño de fragmento más grande cuando aumenta el contenido en níquel y por consiguiente la tenacidad del material. Por consiguiente la relación experimental obtenida entre tamaño de fragmento y velocidad de deformación puede ser expresada una función de tipo potencial dada por:

$\mathrm{L}=\mathrm{L}_{0} \dot{\varepsilon}^{\mathrm{n}}$

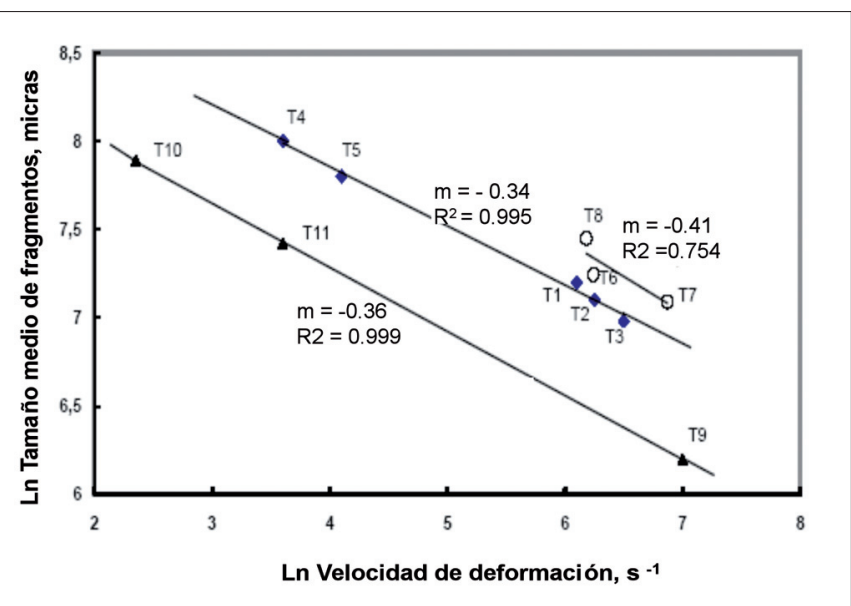

Figura 6. Relación entre tamaño medio de fragmento y velocidad de deformación.

Siendo los valores del exponente, previo ajuste por mínimos cuadrados, de $-0,36+/-0,10$ para alúmina, de $-0,33+/-0,01$ para materiales compuestos de 85 alúmina/15Ni y de $-0,40$ para materiales compuestos de 50 Alumina-50Ni. Como puede apreciarse que los valores experimentales encontrados son más bajos que los predichos por el modelo de escalado $2 / 3$ de Grady (ecuación 13). Similares relaciones potenciales a las de ecuación 15 entre tamaño medio de fragmento y velocidad de deformación con valores inferiores a $-2 / 3$ han sido también obtenidas por otros autores, los cuales han utilizado modelos cohesivos y computacionales de simulación para su explicación [31-33], especialmente a bajas velocidades de deformación. Estos autores han analizado los procesos de fragmentación por métodos numéricos cohesivos indicando que el tamaño medio de fragmento es inferior a los predichos por el modelo 
de Grady, especialmente a bajas velocidades de deformación señalando la importancia de la microestructura previa y los defectos de procesamiento propios del material en el proceso de fragmentación a estas bajas velocidades. Contrariamente a otros modelos teóricos, las simulaciones a meso-escala del modelo de Maiti et al. [32] incorporan el tamaño de grano medio como un parámetro adicional de modelado y asumen que el proceso de fractura tiene lugar de forma inter- granular. En el régimen de alta velocidad, donde la energía cinética domina y una gran cantidad de grietas son simultáneamente activadas dando lugar a una casi instantánea fragmentación de la muestra, Maiti et al. [32] obtuvieron un valor de $\mathrm{n} \approx$ $-0,45$, mientras que el modelo de Drugan [31] predice una similar relación con $n=-0,4264$ Este tipo de relación de tipo potencial ha sido observada también por Curran y Seaman [45] en su modelo numérico basado en micro-mecánica con valores de n de -0,3 a -0,6. A su vez Paliwal y Ramesh [46] también incluyeron un parámetro que captura la sensibilidad de la evolución del deterioro a las características de la distribución de fisuras en el material cerámico. Observaron que mientras que a bajas velocidades de deformación la dispersión en la distribución de fisuras es crítica, a altas velocidades de deformación la densidad de fisuras total es el factor más importante en dictar la respuesta y sensibilidad de los materiales a la velocidad de deformación. Otras investigaciones experimentales han mostrado también que los tamaños de fragmento reales son mucho más pequeños que las estimaciones teóricas establecidas por los modelos energéticos $[47,48]$ a bajas velocidades de deformación, lo que supone aceptar que existen fuentes adicionales de energía, bien la existencia de un trabajo externo, o bien que la energía cinética global deben estar contribuyendo a la creación adicional de superficies de fractura, ya que los modelos de Grady y de Glenn-Chudnovsky solamente asumen la contribución de la energía local al proceso de fragmentación a altas velocidades de deformación. Miller et al. [47] estudiaron el proceso de fragmentación bajo un estado uniforme de velocidad de deformación a través de un marco computacional similar e indicaron la importancia de incorporar el tiempo en el fenómeno de fractura. Señalaron que la discrepancia en el tamaño de fragmento predicho por los modelos de energía a bajas velocidades de deformación radica en asumir por parte de estos modelos de una fractura instantánea. Por tanto, y aunque estos modelos de fragmentación están basados en argumentos simples de conservación de la energía, parecen demasiado simplificados ya que no explican la dinámica de comunicación entre los lugares de grietas o los aspectos estadísticos de las distribuciones de grietas. Las mayores dificultades de estos modelos energéticos proceden, por tanto, de la dificultad para anticipar cuanto de la energía total del sistema contribuye mediante disipación a la creación de superficies fracturadas. Esto ha conducido, dada la interacción compleja de múltiples grietas en el proceso de fractura dinámica y a su dificultad para abordarlo analíticamente, a utilizar modelos computacionales, generalmente de tipo cohesivo, que incorporan el factor tiempo para modelar de forma más correcta y menos simplificada el proceso dinámico de la fragmentación [31-34]. Además ha sido mostrado experimentalmente [49] que mientras la resistencia compresiva cerámica uniaxial es relativamente insensible a velocidades de carga por debajo de $200 \mathrm{~s}^{-1}$ (velocidad de deformación critica), por encima de ese límite aproximado, y especialmente a partir de $1000 \mathrm{~s}^{-1}$, aparece una transición a un régimen de rápido fortalecimiento mecánico con la velocidad de deformación por lo que la resistencia última de fractura (impacto o pico de resistencia) aumenta dramáticamente con la velocidad de deformación aplicada inicialmente. Esto ha sido atribuido a un crecimiento dinámico de grieta dominado por la inercia a partir de grietas pre-existentes [49] De acuerdo con las experiencias reseñadas con anterioridad, esta discrepancia de los composites de alúmina-níquel con el factor de escala $-2 / 3$ del modelo de Grady puede ser debida a la densidad y distribución de fisuras y defectos propios inherentes en estos materiales que actúan sobre el valor del parámetro de escalado $\mathrm{n}$ y a las relativamente bajas velocidades de deformación empleadas en los ensayos.

\subsection{Mecanismos de fractura a alta velocidad de deformación. Análisis micro estructural}

Investigaciones previas [50] en materiales compuestos de matriz cerámica reforzados con fases aisladas dúctiles metálicas han mostrado en ensayos cuasi-estáticos una resistencia al crecimiento de grieta y una cada vez menor sensibilidad de la resistencia a la carga de penetración Vickers (tamaño de fisura) a medida que aumentaba el volumen y el tamaño de grano de molibdeno. Este comportamiento curva $\mathrm{R}$ es atribuido a las fuerzas de anclaje aplicadas por los ligamentos de metal que puentean las caras de grieta más allá del frente de grieta. Este mecanismo de fortalecimiento requiere un enlace fuerte metal cerámico y alta ductilidad de las partículas metálicas. Sin embargo, como puede apreciarse en los resultados de fragmentación anteriores, cuando estos materiales compuestos de alúminaníquel son sometidos a relativamente altas velocidades de deformación, producidas por compresión uniaxial en Barra Hopkinson, se produce un proceso de nucleación inducida por poros y defectos preexistentes generados en el proceso de preparación que conducen a un crecimiento y una coalescencia de multitud de micro grietas orientadas axialmente y a una fragmentación columnar [25] en la dirección de compresión. Como puede apreciarse en la Figura 7, donde se muestran las correspondientes superficies de fractura post mortem de composites de 85 Alúmina/15Ni fragmentados sometidos a altas velocidades de deformación, el mecanismo de despegue metal-cerámico inducido es dominante y no se produce puenteo de grieta por la deformación plástica de las partículas

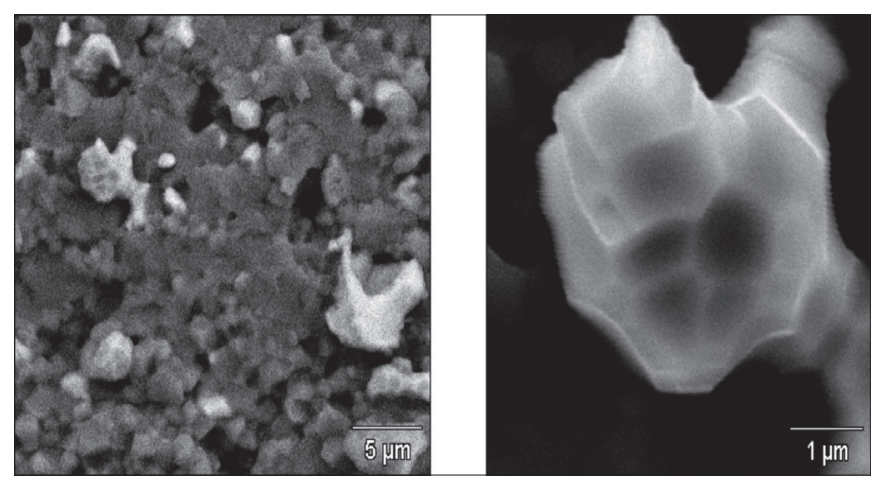

Figura 7. Microscopía electrónica de barrido (MEB) de superficies de fractura post mortem de compuestos de 85 alúmina- $15 \mathrm{Ni}$ sometidos a fractura dinámica a altas velocidades de deformación $\left(\varepsilon *=654 \mathrm{~s}^{-1}\right.$. 
metálicas. Esto significa que el comportamiento de curva $\mathrm{R}$ en ensayos cuasi estáticos es irrelevante en estos procesos de fractura múltiple a altas velocidades de deformación. Como puede apreciarse, aparece una superficie rugosa (7a) donde la mayoría de los granos de níquel (fases brillantes) están desunidos de los granos de alúmina creando huellas o e impresiones permanentes de partículas níquel en las matrices de alúmina (7b). Esto significa que las partículas de níquel apenas experimentan procesos de deformación plástica. Sin embargo el balance energético durante el proceso de fractura indica que parte de la energía cinética del impacto es absorbida por las partículas de níquel dejando menor energía de deformación elástica para su conversión en energía de fractura. Esto explica el mayor tamaño de fragmento producido en la fragmentación dinámica cuando aumenta el contenido en níquel y por tanto la tenacidad de los composites. Puesto que esta energía no es usada en deformar dúctilmente las partículas de níquel pudiera emplearse bien en el despegue de la interfase alúmina-níquel o en un proceso de enroramiento de grieta. Tal mecanismo de endurecimiento por apantallado del frente de grieta ha sido observado por otros investigadores en otros materiales compuestos metal cerámicos [51].

\section{CONCLUSIONES}

Varias conclusiones pueden ser extraídas del comportamiento de materiales compuestos alúmina-níquel cuando son sometidos a altas velocidades de deformación en barra de Hopkinson:

a) El grado de fragmentación y el tamaño medio de fragmento bajo cargas compresivas uniaxiales de alta velocidad depende de la composición y naturaleza del material compuesto (y por lo tanto de su tenacidad), de la velocidad de deformación y de la microestructura y densidad.

b) El análisis estadístico de fragmentos producidos en el impacto, aplicando técnicas de tamizado, muestra que los resultados se ajustan a una distribución de Weibull y que la ecuación exponencial de Rosin y Rammler [27] proporciona una adecuada ajuste de los datos experimentales para un valor del exponente $n$ entre 1.3 y 1.5. Dos regímenes de distribución eran observados con dos distintos parámetros de escala de la distribución, $\lambda_{1}$ y $\lambda_{2}$, lo que corresponde a una región más craqueada y otra más pulverulenta. Estos resultados son consistentes con la teoría de fragmentación de no- equilibrio de Grady [39] para materiales frágiles, que considera que los materiales frágiles tienden a sufrir rotura y fragmentación a una energía de deformación elástica elevada, mayor que la predicha por los métodos cuasi estáticos debido a un proceso de acumulación de energía y a su proceso de liberación temporal en cascada, lo que da lugar a dos parámetros de escala. También están en concordancia con las diferentes regiones de fractura observadas experimentalmente por los materiales frágiles cuando se someten a impactos de alta velocidad donde se aprecian una zona pulverulenta y una zona de grandes fragmentos. Mientras que bajo carga estática, una grieta simple domina el proceso de fractura, bajo cargas dinámicas compresivas como ocurre en la barra Hopkinson, se activan y crecen multitud de grietas inducidas, originan coalescencia y dan lugar finalmente a la correspondiente fragmentación múltiple en la dirección axial de máxima compresión.

c) La influencia de la velocidad de deformación sobre el tamaño medio de fragmento muestra una relación potencial del tipo $\mathrm{L}=$ a. $\varepsilon^{-\mathrm{n}}$ donde los valores de $\mathrm{n}$ experimentales oscilan entre $-0,36 \pm 0,10$ para alúmina, de $-0,33 \pm 0,01$ para materiales compuestos de 85 alúmina/ $15 \mathrm{Ni}$ y de $-0,40$ para materiales compuestos de 50 Alumina- $50 \mathrm{Ni}$. Estos valores son menores que la ley de escala $-2 / 3$ establecida por el modelo energético de Grady [28-29] lo que indica una menor sensibilidad de la fragmentación a los efectos de la velocidad. Similares relaciones potenciales con valores inferiores a $-2 / 3$ han sido también obtenidas por otros autores [31-33]. Esta discrepancia con el factor de escala -2/3 del modelo de Grady puede ser debida a la densidad y distribución de fisuras y defectos propios inherentes en estos materiales que actúan sobre el valor del parámetro $\mathrm{n}$ y al rango de velocidades sobre los que se realizan los ensayos. Una explicación de tales desviaciones puede encontrarse también en el modelo energético propuesto por Glenn and Chudnovksy (modelo GC) [30] quienes propusieron una nueva ecuación para el tamaño medio de fragmento introduciendo un término de corrección en el modelo de Grady que contempla la energía elástica de deformación almacenada antes de la fractura así como la existencia de un pico de tensión $\sigma_{\text {máx. }}$ a partir del cual se inicia la fragmentación. Debido a estas correcciones, este modelo GC predice unos menores valores del tamaño de fragmento y una menor sensibilidad a bajas velocidades de deformación.

d) El análisis microestructural de los fragmentos observado por microscopía electrónica después del proceso de fragmentación muestra que las partículas de níquel están completamente despegados de la matriz de alúmina sin apenas deformación plástica. A pesar de las diferentes velocidades de impacto compresivo aplicadas se observaron similares patrones de deterioro. A altas velocidades de deformación las partículas de Ni se desligan de la matriz de alúmina frágil dejando las correspondientes huellas (imprints). Estos huecos dejados por las partículas de níquel se producen durante el proceso de sinterización que da lugar a un crecimiento de grano y reducción de la porosidad. Sin embargo, parte de la energía cinética de impacto es absorbida bien por las partículas de níquel o pudiera emplearse en un proceso de embotamiento de grieta reduciendo así su proceso de nucleación haciendo que el tamaño de fragmento crezca con el contenido en níquel $\mathrm{y}$, por lo tanto, con la tenacidad de los material compuestos alúmina-níquel estudiados.

\section{BIBLIOGRAFÍA}

1. Trumble, K. P.; Ruhle, M., (1991): The thermodynamics of spinel interphase formation at diffusion-bonded Ni/Al2O3 interfaces, Acta Metall. Mater., 39(8):1915-19242. http:/ / dx.doi.org/10.1016/0956-7151(91)90160-3

2. Sekino, T.; Nakajima, T.; Ueda, S.; Nihara, K., (1997): Reduction and sintering of a nickel-dispersed-alumina composite and its properties, J. Am. Ceram. Soc., 80(5): 1139-1148. http:/ / dx.doi.org/10.1111/j.1151-2916.1997. tb02956.x

3. Tuan, W. H. and Brook, R. J., (1990): The toughening of alumina with nickel inclusions, J. Eur. Ceram. Soc., 6(1): 31-37. http: / / dx.doi.org/10.1016/09552219(90)90032-B 
4. Rodeghiero E. D; Tse, O. K.; Chisake J.; Giannelis E. P., (1995): Synthesis and Properties of Ni-a-Al2O3 Composites via Sol-Gel, Mater. Sci. Eng. A, 195: 151-61. http: / / dx.doi.org/10.1016/0921-5093(94)06514-4

5. Breval E.; Deng Z.; Shiou S.; Pantano, C.G., (1992): Sol-Gel Prepared Ni-Alumina Composite Materials, Part I: Microstructure and Mechanical Properties, J. Mater. Sci., 27: 1464-68. http://dx.doi.org/10.1007/ BF00542904

6. Tuan, W.H.; Lin, M.C.; Wu, H.H., (1995):Preparation of Al2O3/Ni Composites by Pressureless Sintering in H2, Ceram. Int., 21: 221-25. http:/ / dx.doi.org/10.1016/0272-8842(95)99785-A

7. Tuan, W.H.; Brook, R.J., (1992): Processing of Alumina/ Nickel Composites, J. Eur. Ceram. Soc., 10: 95-100. http://dx.doi.org/10.1016/09552219(92)90123-U

8. Stundag, E.U.; Ret, P.; Subramanian, R.; Dieckmann, R.; Sass, S.L., (1995): In Situ Metal-Ceramic Microstructures by Partial Reduction Reactions in the Ni-Al-O System and the Role of ZrO2, Mater. Sci. Eng. A, 195: 39-50. http: / / dx.doi.org/10.1016/0921-5093(94)06504-7

9. Jones, S.A.; Burlitch, J.M.; Stündag, E.U.; Yoo, J.; Zehnder, A.T., (1995): Nickel-Alumina Composites: In Situ Synthesis by a Displacement Reaction and Mechanical Properties; pp. 53-58 in Materials Research Society Symposium Proceedings, Vol. 365, Ceramic Matrix Composites-Advanced High-Temperature Materials. Edited by R. A. Lowden, M. K. Ferber, J. R. Hellmann, K. K. Chawla, and S. G. DiPietro. Materials Research Society, Pittsburgh, PA.

10. Fahrenholtz, W. G.; Ewsuk, K. G.; Loehman, R. E.; Tomsia A. P., (1996): Formation of Structural Intermetallics by Reactive Metal Penetration of Ti and Ni Oxides and Aluminates, Metall. Mater. Trans. A, 27A [8]: 2122-29.

11. Schicker, S.; García, D. E.; Bruhn, J.; Janssen, R.; Claussen, N., (1998): Reaction Synthesized Al2O3-Based Intermetallic Composites, Acta Mater. 46 [7]: 2485-92. http: / / dx.doi.org/10.1016/S1359-6454(98)80032-3

12. Antolin, S.; Nagelberg, A. S.; Creber, D. K., (1992): Formation of Al2O3/ Metal Composites by the Directed Oxidation of Molten AluminumMagnesium-Silicon Alloys: I, Microstructural Development, J. Am. Ceram. Soc., 75 [2]: 447-54. http:/ / dx.doi.org/10.1111/j.1151-2916.1992. tb08200.x

13. Hernandez, N.; Moreno, R.; Sanchez-Herencia, A. J.; Fierro, J. L. G., (2005) Surface Behavior of Nickel Powders in Aqueous Suspensions, J. Phys. Chem. B, 109 [10]: 4470-4. http:/ / dx.doi.org/10.1021/jp0448954

14. Hernandez, N.; Sanchez-Herencia, A. J.; Moreno R., (2005): Forming of Nickel Compacts by a Colloidal Filtration Route, Acta Mater. 53 [4]: 919-25. http: / / dx.doi.org/10.1016/j.actamat. 2004.10.038

15. Sanchez- Herencia, A. J.; Hernandez, N.; Moreno, R., (2006): Rheological Behavior and Slip Casting of Al2O3-Ni Aqueous Suspensions, J. Am. Ceram. Soc., 89 [6]: 1890-1896. http://dx.doi.org/10.1111/j.15512916.2006.01045.x

16. Tseng, W. J.; Chen, C. N., (2002): Determination of maximum solids concentration in nickel nanoparticle suspensions, J. Mater. Sci. Lett. 21(5): 419-422. http: / / dx.doi.org/10.1023/ A:1014988008397

17. Tseng, W. J.; Chen, C. N., (2003): Effect of polymeric dispersant on rheological behavior of nickel-terpineol suspensions, Mater. Sci. Eng A 347: 145-153. http:/ / dx.doi.org/10.1016/S0921-5093(02)00562-2

18. Sun, X.; Yeohmans, J., (1996): Optimization of a Ductile-ParticleToughened Ceramic, J. Am. Ceram. Soc., 79 [10]: 2705-17. http://dx.doi. org /10.1111/j.1151-2916.1996.tb09036.x

19. Fahrenholtz, W.G.; Ellerby, D. T.; Loehman, R. E., (2000): Al2O3-Ni Composites with High Strength and Fracture Toughness, J. Am. Ceram. Soc., 83 [5]: 1279-80. http:/ / dx.doi.org/10.1111/j.1151-2916.2000.tb01368.x

20. Meyers, M.A., (1994): Dynamic Behaviour of Materials. Wiley, New York http:/ / dx.doi.org/10.1002/9780470172278

21. Frew, D. J.; Forrester, M.J.; Chen. W., (2002): Pulse shaping techniques for testing brittle materials with a split Hopkinson pressure bar, Experimental Mechanics. 93-106. http:/ / dx.doi.org/10.1007/BF02411056

22. Nemat-Nasser, S.; Isaacs, J. B.; Starrett, J. E., (1991): Hopkinson Techniques for Dynamic Recovery Experiments, Proc. Royal Soc. Lond, A435: 371-91. http: / / dx.doi.org/10.1098/rspa.1991.0150

23. Subhash, G.; Maiti, S.; Geubelle, P.; Ghosh. D., (2008): Recent advances in dynamic indentation fracture, impact damage and fragmentation of ceramics, J. Am. Ceram. Soc. 91, (9): 2777-91. http://dx.doi.org/10.1111/ j.1551-2916.2008.02624.x

24. Lankford, J., (1981): Mechanisms Responsible for Strain-Rate-Dependent Compressive Strength in Ceramic Materials, J. Am. Ceram. Soc, 64 [2] C-33-C-34. http:/ / dx.doi.org/10.1111/j.1151-2916.1981.tb09570.x

25. Lankford, J., (1991): Fragmentation of brittle materials at high rates of loading, J. of Mat. Sci. 26: 745-750. http:/ / dx.doi.org/10.1007/BF01124844

26. Mott, N.F., (1947): Fragmentation of shell cases, Proceedings of the Royal Society of London, Series A (Mathematical and Physical Sciences). 300-308.

27. Rosin, P.; Rammler, E., (1933): The laws governing the fineness of powdered coal, J Inst Fuel. 29-36.
28. Grady, D.E., (1982): Local Inertial Effects in Dynamic Fragmentation, J. Appl. Phys., 53 [1] : 322-25. http:/ / dx.doi.org/10.1063/1.329934

29. Kipp, M.E.; Grady, D.E., (1985): Dynamic fracture growth and interaction in one dimension, Journal of Mechanics and Physics of Solids. 33, (4): 399415. http: / / dx.doi.org/10.1016/0022-5096(85)90036-5

30. Glenn, L.A.; and Chudnovsky, A., (1986): Strain-Energy Effects on Dynamic Fragmentation, J. Appl. Phys., 59 [4]: 1379-80. http://dx.doi. org $/ 10.1063 / 1.336532$

31. Drugan W.J., (2001): Dynamic fragmentation of brittle materials: analytical mechanics-based models, J Mech Phys Solid 49: 1181-1208. http:/ / dx.doi. org/10.1016/S0022-5096(01)00002-3

32. Maiti, S.; Rangaswamy, K.; Geubelle, P. H., (2005): Mesoscale analysis of dynamic fragmentation of ceramics under tension, Acta Materialia 53: 823-834. http:/ / dx.doi.org/10.1016/j.actamat.2004.10.034

33. Zhou, F.; Molinari, J.; Ramesh, K. T., (2005): A Cohesive Model Based Fragmentation Analysis: Effects of Strain Rate and Initial Defects Distribution, Int. J. Solids Struct.42: 5181-207. http: / / dx.doi.org/10.1016/j. ijsolstr.2005.02.009

34. Yu, R. C.; Ruiz, G.; Pandolfi, A., (2004): Numerical Investigation on the Dynamic Behaviour of Advanced Ceramics, Eng. Fracture Mech., 71: 897-911. http: / / dx.doi.org/10.1016/S0013-7944(03)00016-X

35. Song, B.; Chen, W., (2005): Split Hopkinson pressure bar techniques for characterizing soft materials, Latin American Journal of Solids and Structures 2: 113-152

36. Ravichandran, G.; Subash. G., (1994): Critical appraisal of limiting strain rates for compression testing of ceramics in a split Hopkinson pressure bar, J. Am. Ceram. Soc. 77: 263-67. http:/ / dx.doi.org/10.1111/j.1151-2916.1994. tb06987.x

37. Martinez, M.A.; Chocron, I.S.; Rodriguez, J.; Sánchez Gálvez, V.; Sastre, L.A., (1998): Confined compression of elactic adhesives at high rates of strain, International Journal of Adhesion and adhesives 18: 375-83. http:/ / dx.doi.org/10.1016/S0143-7496(98)00023-2

38. Orgaz, F.; Gómez del Río, T.; Varela, A.; Fernández Lozano, J.F., (2010): Dynamic behavior of thick alumina layers with tunnelled interfaces. Design, development, and applications of engineering ceramics and composites, Ceramic Transactions 215: 27-40.

39. Grady, D.E., (2010): Length scales and size distributions in dynamic fragmentation, Int. J. Fract 163, (1-2): 85-99. http://dx.doi.org/10.1007/ s10704-009-9418-4

40. Lienau, C. C., (1936): Random Fracture of a Brittle Solid, J. Franklin Inst. 221 [1324]: 485-94. http:/ / dx.doi.org/10.1016/S0016-0032(36)90309-5

41. Grady, D. E.; Kipp, M. E., (1985): Geometric Statistics and Dynamics Fragmentation, J. Appl. Phys., 58: 1210-22. http://dx.doi. org / 10.1063/1.336139

42. Grady, D. E.; Kipp, M. E., (1985): Mechanisms of Dynamic Fragmentation: Factors Governing Fragment Size, Mech. Mater., 4: 311-24. http:/ / dx.doi. org/10.1016/0167-6636(85)90028-6

43. Mott, N. F.; Linfoot, E. H., (1943): Ministry of Supply, AC 3348.

44. Grady, D. E., (1990): Particle Size Statistics in Dynamic Fragmentation, J. Appl. Phys., 68: 6099-105. http:/ / dx.doi.org/10.1063/1.347188

45. Curran, D.; Seaman, L.; Cooper, T.; Shockey, D., (1990): Micromechanical model for comminution and granular flow of brittle material under high strain rate application to penetration of ceramic targets, Int. J. Impact Eng. 13: 53-83. http: / / dx.doi.org/10.1016/0734-743X(93)90108-J

46. Paliwal, B.; Ramesh, K. T., (2008): An Interacting Micro-Crack Damage Model for Failure of Brittle Materials under Compression, J. Mech. Phys. Solids, 56: 896-923. http:/ / dx.doi.org/10.1016/j.jmps.2007.06.012

47. Miller, O.; Freund, L. B.; Needleman, A., (1999): Modelling and Simulation of Dynamic Fragmentation in Brittle Materials, Int. J. Fract., 96: 101-215. http: / / dx.doi.org/10.1023/ A:1018666317448

48. Wang, H.; Ramesh, K.T., (2004): Dynamical strength and fragmentation of hot-pressed silicon carbide under uniaxial compression, Acta Materialia 52: 355-367. http:/ / dx.doi.org/10.1016/j.actamat.2003.09.036

49. Lankford, J., (1991): Fragmentation of brittle materials at high rates of loading, J. of Mat. Sci., 26: 745-750.

50. Bartolomé, J. F.; Díaz, M.; Requena, J.; Moya, J. S., (2002): Influence of the Metal Particle Size on the Crack Growth Resistance in Mullite/ Molybdenum Composites, J. Am. Ceram. Soc. 85 (11): 2778-2784. http:// dx.doi.org/10.1111/j.1151-2916.2002.tb00528.x

51. Bencher, C.D.; Sakaida, A.; Venkateswara Rao, K.T; Ritchie, R.O., (1995): Toughening mechanisms in ductile niobium-reinforced niobium aluminide (Nb/Nb3Al)in situ composites, Metall. Mater. Trans. A 26: 2027-2033. http:/ / dx.doi.org/10.1007/BF02670674

Recibido: 10/06/2014

Recibida versión corregida: 14/10/2014

Aceptado: 15/10/2014 